Sección Agrícola / Agriculture

Artículos de investigación / Research paper

\title{
Preferencias de tisanópteros (Thysanoptera) por las estructuras aéreas de las plantas de algodón (Gossypium hirsutum) en Colombia
}

\author{
Preferences of thrips (Thysanoptera) for the aerial structures of cotton plants (Gossypium hirsutum) in Colombia \\ CAMILO IGNACIO JARAMILLO-BARRIOS ${ }^{1}$, ANDRÉS RODRÍGUEZ², EDGAR VARÓN-DEVIA³, \\ BUENAVENTURA MONJE-ANDRADE ${ }^{4}$ y EVERTH EBRATT-RAVELO 5
}

\begin{abstract}
Resumen: El objetivo del presente estudio fue determinar los niveles de preferencia de especies de trips por estructuras aéreas de las plantas de algodón. Para ello, en el valle cálido del alto Magdalena en la región Andina de Colombia, se coleccionaron e identificaron especies de trips asociadas al sistema productivo de algodón. Se muestrearon 60 fincas productoras en fase de llenado de cápsulas, donde la planta de algodón presentó los microhábitats: hoja joven, botón floral, flor y cápsula. Se realizaron análisis de agrupamiento de Bray-Curtis y pruebas no paramétricas de KruskallWallis. Las especies registradas fueron Thrips palmi, Scirtothrips dorsalis y Frankliniella cephalica. Thrips palmi (n $=3595 ; \mu=14,97 \pm 32,35)$ fue la más frecuente, seguida por $S$. dorsalis $(\mathrm{n}=856 ; \mu=3,56 \pm 6,35)$ y Fr. cephalica $(\mathrm{n}=405 ; \mu=1,68 \pm 9,13)$. Thrips palmi se encontró en todos los microhábitats; $S$. dorsalis prefirió cápsula, seguido de hoja joven y botón floral. Frankliniella cephalica mostró preferencia por las flores. El presente estudio reveló la acción fitófaga de T. palmi y S. dorsalis como insectos con preferencia intra-hospedante del tipo generalista, hacia hojas (filofagía), botones florales (antofagía) y cápsulas (carpofagía). Frankliniella cephalica se comportó como especialista en flores (antofagía) de algodón.
\end{abstract}

Palabras clave: Hospedante, trips, fitófago, Malvaceae.

\begin{abstract}
The aim of this study was to determine the preference levels of species of thrips for aerial structures of cotton plants. To accomplish this, samples of associated thrips species to cotton crop were collected and identified in warm weather valleys of the high Magdalena, in the Andean region of Colombia, A total of 60 cotton producing farms were sampled during the capsule filling phase in which the cotton plants presented young leaf, flower bud, flower and capsule microhabitats. The Bray-Curtis Cluster analysis and the Kruskall-Wallis non-parametric test were utilized. The thrips species found were: Thrips palmi, Scirtothrips dorsalis, and Frankliniella cephalica. T. palmi was the most frequent (n $=3595 ; \mu=14.97 \pm 32.35)$, while $S$. dorsalis $(\mathrm{n}=856 ; \mu=3.56 \pm 6.35)$ and $F r$. cephalica $(\mathrm{n}=405 ; \mu=1.68 \pm 9.13)$ were found in lower numbers. Thrips palmi was found in all four micro-habitats, while $S$. dorsalis preferred capsules, followed by leaves and floral buds. Frankliniella cephalica showed preference for flowers. The present study revealed the phytophagous action of $T$. palmi and $S$. dorsalis as insects with intra-host generalist behavior, with preferences for leaves (phyllophagous), flower buds (anthophagous) and capsules (carpophagous). Frankliniella cephalica acted as a specialist, with a preference for the flowers (anthophagous) of the cotton plant.
\end{abstract}

Key words: Hosts, thrips, phytophagous, Malvaceae.

\section{Introducción}

Los insectos fitófagos detectan partes muy diversas de las plantas en búsqueda del recurso alimenticio. De acuerdo con la estrategia, se ha realizado una clasificación general; los polífagos, que son insectos que se alimentan de plantas de diferentes familias; oligófagos, especies asociadas a diferentes géneros dentro de una misma familia; estenófagos, insectos que se hospedan en especies del mismo género y monófagos, los cuales se encuentran asociados a una sola especie vegetal hospedante. En relación con la estructura de la planta, el patrón de explotación puede ser por antófagia (consumo de estructuras florales), carpofagia, (alimentación en frutos) o filofagia (daños en hojas). Según la amplitud de dicha relación los insectos fitófagos pueden ser especialistas o generalistas (Zwolfer 1983; Pérez-Contreras 1999; Marullo 2009; Zamar y Neder 2012).

Los trips (Thysanoptera: Thripidae), son insectos que presentan notables cambios en los hospedantes, de tal manera que se les puede encontrar en plantas ajenas a sus anfitriones "naturales", ya sea como fuente alimenticia o de reposo, varios autores se refieren a estas plantas como lugares de paso para los trips (Mound y Marullo 1996); sin embargo, la especialización en los trips podría corresponder a la regla general y no a la excepción (Pérez-Contreras 1999).

\footnotetext{
${ }^{1}$ Ingeniero Agrónomo, Corporación Colombiana de Investigación Agropecuaria-Agrosavia, Centro de Investigación Nataima Km 9 vía Espinal-Chicoral, Tolima, Colombia, cijaramillo@agrosavia.co. Código Orcid: https://orcid.org/0000-0002-8302-2736. ${ }^{2}$ Estudiante, Facultad de Agronomía. Universidad del Tolima, Ibagué, Colombia, ing.andresrz@gmail.com. ${ }^{3} \mathrm{Ph}$. D. Corporación Colombiana de Investigación Agropecuaria-Agrosavia, Centro de Investigación Nataima Km 9 vía Espinal-Chicoral, Tolima, Colombia, evaron@agrosavia.co. Código Orcid: https://orcid.org/0000-0002-5421-507X. ${ }^{4}$ M. Sc. Corporación Colombiana de Investigación Agropecuaria-Agrosavia, Centro de Investigación Nataima Km 9 vía Espinal-Chicoral, Tolima, Colombia, bmonje@agrosavia. co. Código Orcid: http://orcid.org/0000-0002-8177-4651. ${ }^{5}$ I. A., M. Sc. Candidato Ph. D. Universidad Nacional de Colombia. Instituto Colombiano Agropecuario ICA. Mosquera, Cundinamarca, Colombia, eeebraitr@unal.edu.co. Autor para correspondencia. Camilo Ignacio Jaramillo-Barrios. Ingeniero Agrónomo, Corporación Colombiana de Investigación Agropecuaria-Agrosavia, Centro de Investigación Nataima Km 9 vía Espinal-Chicoral, Tolima, Colombia. cijaramillo@agrosavia.co.
} 
Con más de 2.100 especies, Thripidae, es la segunda familia más grande de Thysanoptera. Aunque a veces se considera que son principalmente antófilas, una gran proporción de especies se reproduce solo en hojas, algunos son depredadoras de otros artrópodos pequeños, y muy pocas se asocian con musgos. La mayoría de los trips plaga y todos los vectores de Tospovirus son miembros de esta familia (Jones 2005; ThripsWiki 2018).

En Colombia el cultivo de algodón es importante desde el punto de vista social y económico. En el valle cálido del alto Magdalena se encuentran las principales localidades productoras con aproximadamente 9.720 ha y rendimientos promedio de $1.135 \mathrm{~kg}$ de fibra por ha (Confederación Colombiana del Algodón 2015).

Al presente, las principales plagas registradas en el cultivo de algodón son el picudo del algodonero (Anthonomus grandis Boheman), complejo Spodoptera spp., gusano bellotero (Heliothis virescens Fabricius), mosca blanca (Bemisia tabaci Gennadius), entre otras. En Colombia, se han realizado estudios para determinar el potencial de daño de A. grandis, la evaluación del complejo Spodoptera tras la introducción del algodón transgénico, la susceptibilidad de $H$. virescens a la proteína Cry1Ac y se han implementado estrategias de manejo con formulados para el control de $B$. tabacci en condiciones de laboratorio (Martínez et al. 2003; Espinel et al. 2008; Santos-Amaya et al. 2009; Romero et al. 2012).

Ahora bien, en Colombia los thrips han sido importantes para estudios de determinación de umbrales de acción, niveles de daño económico y estrategias de manejo en hortalizas, flores y frutales como en banano y maracuyá (Arévalo y Correa 2003; Bueno y Cardona 2003; Santos et al. 2012; Sarabia et al. 2015). En algodón, algunas especies de thrips (Thysanoptera: Thripidae), han adquirido mayor relevancia económica por la capacidad de ocasionar daños directos como la distorsión de hojas, defoliación, muerte de meristemas apicales, aborto de botones florales, bronceado de cápsulas, apertura prematura de cápsulas y daños indirectos por la transmisión de agentes patológicos virales que afectan la producción y calidad de la fibra (EFSA 2014).

Por lo anterior, el presente trabajo tuvo como objetivos determinar las especies de Thripidae asociadas al cultivo de algodón y sus preferencias por los microhábitats de las plantas: (hoja, botón floral, flor y cápsula), bajo condiciones ambientales de campo. Esta información servirá de base para optimizar las actividades de muestreo de estos insectos en cultivos de algodón en el valle cálido del alto Magdalena de Colombia.

\section{Materiales y métodos}

Área de estudio. Los muestreos se realizaron en la zona del valle cálido del alto Magdalena, en los departamentos del Huila, Tolima y Cundinamarca (región Andina de Colombia) en la franja altitudinal basal por debajo de $1.000 \mathrm{msnm}$ ubicadas en los municipios de Ambalema, Armero Guayabal, Venadillo, Espinal y Natagaima, en el departamento del Tolima; Villavieja y Campoalegre, en el departamento del Huila y Ricaurte en Cundinamarca.

Muestreos en cultivos de algodón. Durante 2014, se seleccionaron 60 fincas productoras de algodón que presentaron cultivos de algodón en etapa fenológica de llenado de cápsu- las (80 y 100 DDE), con presencia simultánea de hojas jóvenes, botones florales, flores y cápsulas.

En cada finca se muestrearon 10 plantas por ha y se capturaron de forma directa, con ayuda de pinceles humedecidos con alcohol, los trips adultos y larvas que hicieron presencia en cinco hojas jóvenes, cinco botones florales, cinco cápsulas y cinco flores de cada planta. Los trips recolectados se depositaron en tubos eppendorf de 1,5 mL con etanol al $70 \%$.

Fase de laboratorio. Las muestras coleccionadas se trasladaron al laboratorio de entomología del centro de investigación Nataima de Corpoica, allí se realizó la clasificación previa por morfotipos para diferenciar los estados inmaduros y adultos. Los adultos se contabilizaron y mediante montajes semipermanentes en solución Hoyer, se identificaron a nivel de especie de acuerdo con Mound y Marullo (1996), Hoddle y Mound (2003) y Kumar et al. (2013). El análisis y las observaciones de los montajes se realizaron con la ayuda de un estereoscopio marca Leica ZOOM 2000 y un microscopio Nikon Type 119 YS2-T.

Análisis de datos. Con las abundancias de cada especie, se realizaron análisis exploratorios mediante estadística descriptiva en los microhábitats evaluados. Se determinó la relación de cada especie con los microhábitats mediante el análisis de agrupamiento de Bray-Curtis con ligamiento por promedio de grupos y un valor crítico del $70 \%$ de similaridad en el programa Biodiversity Pro (McAleece et al. 1997). Los registros porcentuales de los cálculos de los índices de BrayCurtis se transformaron con el Log10 para obtener los niveles de preferencia de acuerdo con intervalos $(>1)$ de preferencia por el recurso, $(=1)$ presencia accidental en el recurso y $(<1)$ evasión del recurso. Se compararon las abundancias entre estructuras, especies, estructura-especie, departamentoestructura-especie, zona-estructura-especie mediante pruebas no paramétricas de Kruskal-Wallis al $5 \%$ de significancia y se compararon de a pares entre las medias de los rangos con el programa Infostat (Di Rienzo et al. 2016).

\section{Resultados}

A partir de 12.000 muestras, se confirmó la presencia, en orden de abundancia, de T. palmi $(\mathrm{n}=3595 ; \mu=14,97 \pm 2,08)$, $S$. dorsalis $(\mathrm{n}=856 ; \mu=3,56 \pm 0,41)$, y Fr. cephalica $(\mathrm{n}=$ $405 ; \mu=1,68 \pm 0,59$ ) en el cultivo de algodón en los departamentos del Huila, Cundinamarca y Tolima en el valle cálido del alto Magdalena de Colombia.

Las abundancias promedio en los tres departamentos, presentaron diferencias significativas $(\mathrm{H}=160,78 ; \mathrm{P}<0,0001)$. T. palmi, presentó la mayor abundancia en los microhabitats hoja joven, flor, botón floral y cápsula en los departamentos del Huila y Tolima, seguido por $S$. dorsalis en hoja joven en Cundinamarca, flor en el Huila, botón floral en el Tolima y cápsula en Cundinamarca y por Fr. cephalica en hoja joven en Tolima, y flor en Tolima, Huila, Cundinamarca; no hubo presencia de $F r$. cephalica en botón floral y cápsula en el Huila $(\mathrm{H}=13,05 ; \mathrm{P}<0,0015)$ (Figs. 1A-1L).

Thrips palmi estuvo presente en todas las zonas estudiadas, sin embargo, las mayores abundancias se observaron en hojas jóvenes, botones florales y flores en la zona centro del Huila, y en cápsulas en la zona sur del Tolima (Figs. 2A-2D). Scirtothrips dorsalis presentó las mayores abundancias en hojas jóvenes y cápsulas del suroccidente de Cundinamarca, 

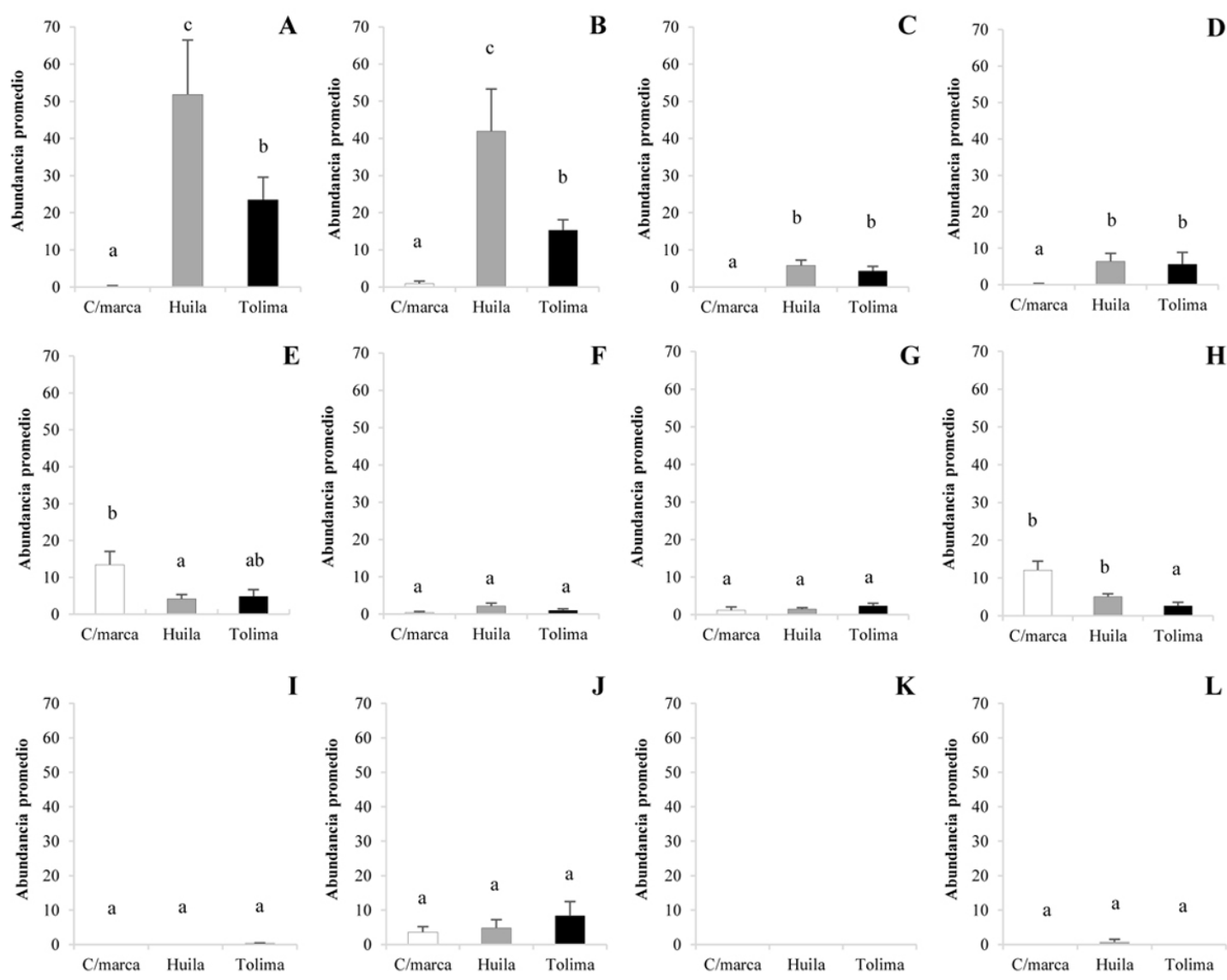

Figura 1. Promedio de abundancia de T. palmi, S. dorsalis y Fr. cephalica ( \pm SE) en microhábitat foliares y reproductivos del cultivo de algodón en los departamentos de Cundinamarca (C/marca), Tolima y Huila. A. T. palmi en hoja joven. B. T. palmi flor. C. T. palmi en botón floral. D. T. palmi en cápsula. E. S. dorsalis en hoja joven. F. S. dorsalis en flor. G. $S$. dorsalis en botón floral. H. S. dorsalis en cápsula. I. Fr. cephalica en hoja joven. J. Fr. cephalica en flor. K. Fr. cephalica en botón floral. L. Fr. cephalica en cápsula (Los promedios con la misma letra, no presentan diferencias significativas-Prueba Kruskal-Wallis, $\mathrm{H}=160,78 ; \mathrm{P}<0,0001)$.

seguido de flor y botón floral en el centro del Huila y sur del Tolima (Figs. 3A-3D). Frankliniella cephalica presentó las mayores abundancias en flor, en el sur de Tolima, norte del Huila y suroccidente de Cundinamarca. Las flores representaron el microhábitat óptimo en todas las zonas algodoneras $(\mathrm{H}=27,92 ; \mathrm{P}<0,0001)$ (Figs. 4A-4D).

Agrupamiento de Bray-Curtis y preferencia por microhábitat. El análisis de agrupamiento de Bray- Curtis entre los microhábitats agrupados (Fig. 5) para T. palmi evidenció dos grupos con índices de similitud (Is) de 0,79 entre botón floral-cápsula y 0,76 entre flor-hoja (Fig. 5A). Los niveles de preferencia fueron: hoja joven $(1,9>1)$, seguido por flor $(1,9$ $>1)$, botón floral $(1,8>1)$ y cápsula $(1,7>1)$.

Scirtothrips dorsalis presentó un índice de similitud (Is) de 0,82 entre hoja-cápsula (Fig. 5B). En cuanto al análisis de preferencia, mostró una mayor predilección por las cápsulas $(1,7>1)$, seguido de hoja $(1,3>1)$ y botón floral $(1,2>1)$.
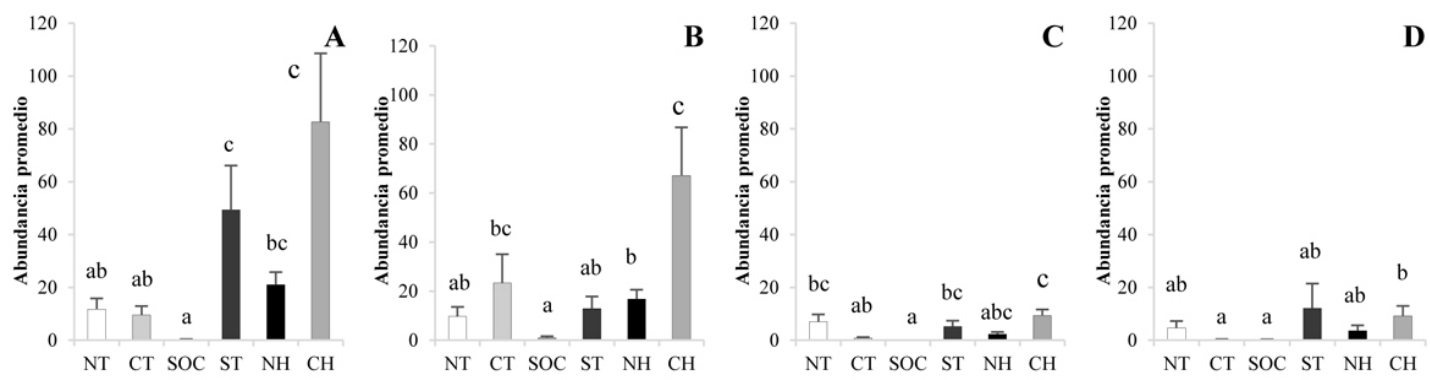

Figura 2. Abundancias de Thrips palmi $( \pm \mathrm{SE})$ en microhábitat foliares y reproductivos en las zonas algodoneras del valle cálido del alto Magdalena, Colombia, norte del Tolima (NT), centro del Tolima (CT), suroccidente de Cundinamarca (SOC), sur del Tolima (ST), norte del Huila (NH), centro del Huila (CH). A. T. palmi hoja joven. B. T. palmi flor. C. T. palmi botón floral. D. T. palmi. Letras iguales no presentan diferencias significativas entre promedios de rangos en zonas algodoneras $(\mathrm{H}=36,84 ; \mathrm{P}<0,0001)$. 

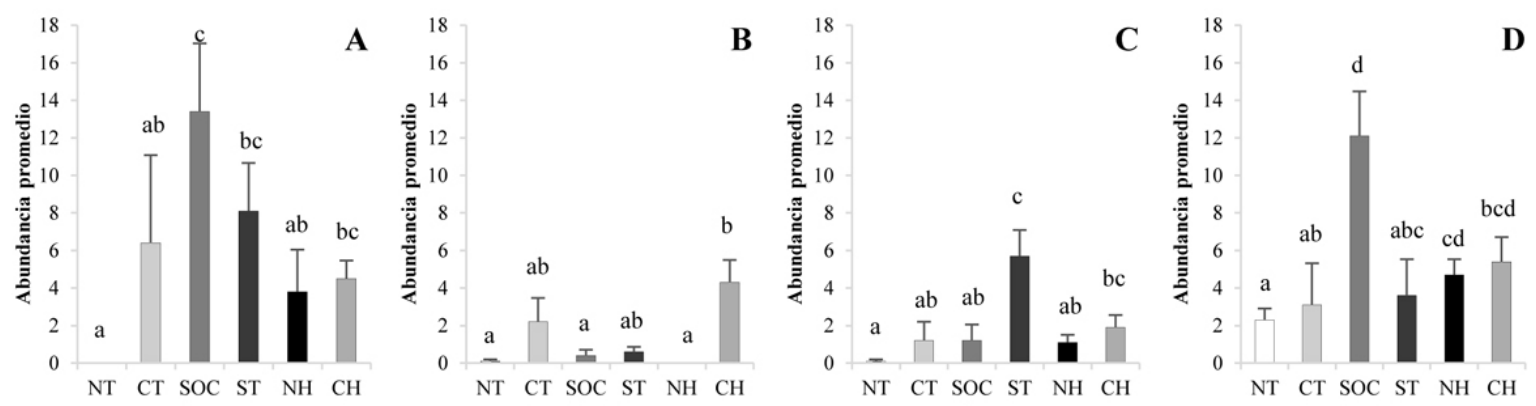

Figura 3. Abundancias de Scirtothrips dorsalis ( \pm SE) en microhábitat foliares y reproductivos en las zonas algodoneras del valle cálido del alto Magdalena, Colombia, norte del Tolima (NT), centro del Tolima (CT), suroccidente de Cundinamarca (SOC), sur del Tolima (ST), norte del Huila (NH), centro del Huila (CH). A. S. dorsalis hoja joven. B. S. dorsalis flor. C. S. dorsalis botón floral. D. $S$. dorsalis cápsula. Letras iguales no presentan diferencias significativas entre promedios de rangos en zonas algodoneras $(\mathrm{H}=$ $19,65 ; \mathrm{P}<0,0001)$.

El microhábitat flor $(0,8<1)$ correspondió a una preferencia baja o prescindible por el insecto.

Frankliniella cephalica no evidenció agrupamiento, demostrando que existe una respuesta negativa hacia cada uno de los microhábitats en las plantas de algodón (Fig. 5C). Esta especie mostró preferencia por la flor $(1,3>1)$. Las cápsulas $(0,4<1)$, hojas $(-0.4<1)$ y botones florales no presentaron individuos para esta especie). La comparación entre estructuras arrojó diferencias significativas para T. palmi $(\mathrm{H}=36,84$; $\mathrm{P}<0,0001)$, S. dorsalis $(\mathrm{H}=19,65 ; \mathrm{P}<0,0001)$ y Fr. cephalica $(\mathrm{H}=31,16 ; \mathrm{P}<0,0001)$ (Tabla 1).

Al analizar la interacción entre las tres especies, se observa que cada microhábitat es compartido por al menos dos de las tres especies de trips asociados al cultivo de algodón
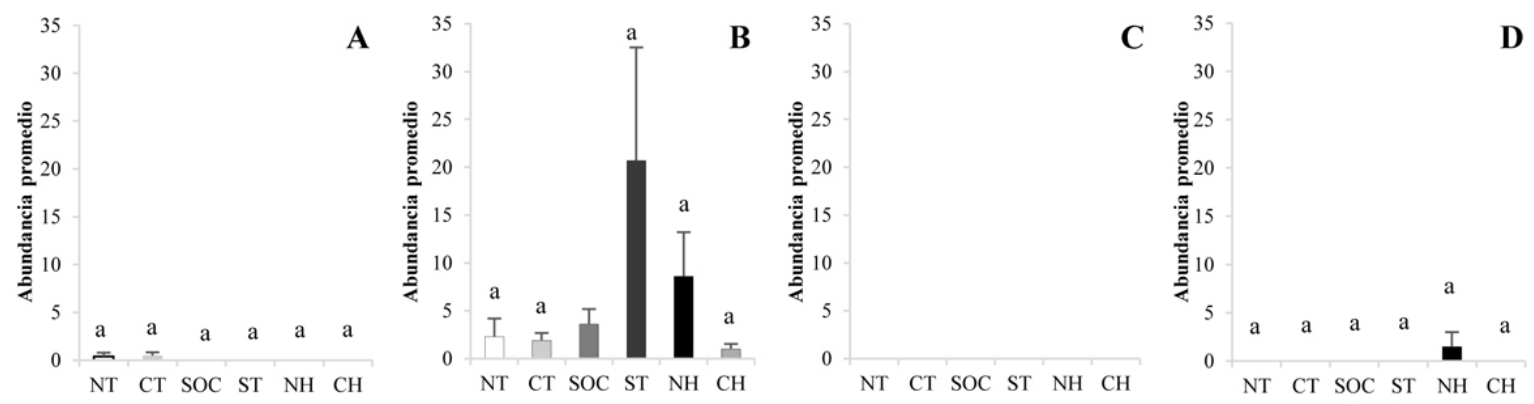

Figura 4. Abundancias de Fr. cephalica $( \pm \mathrm{SE})$ en microhábitat foliares y reproductivos en las zonas algodoneras del valle cálido del alto Magdalena, Colombia, norte del Tolima (NT), centro del Tolima (CT), suroccidente de Cundinamarca (SOC), sur del Tolima (ST), norte del Huila (NH), centro del Huila (CH). A. Fr. cephalica hoja joven. B. Fr. cephalica flor. C. Fr. cephalica botón floral. D. Fr. cephalica cápsula. Letras iguales no presentan diferencias significativas entre promedios de rangos en zonas algodoneras $(\mathrm{H}$ $=31,16 ; \mathrm{P}<0,0001)$.

Tabla 1. Abundancias ( \pm SE) para T. palmi, S. dorsalis y Fr. cephalica en hoja joven, flor, botón floral y cápsula del cultivo de algodón en el valle cálido del alto Magdalena.

\begin{tabular}{lccc}
\hline \multicolumn{1}{c}{ Microhábitat } & \multicolumn{1}{c}{ T. palmi } & S. dorsalis & Fr. cephalica \\
\hline Hoja & $29,05 \pm 6,27^{\mathrm{b}}$ & $6,03 \pm 1,22^{\mathrm{b}}$ & $0,15 \pm 0,09^{\mathrm{a}}$ \\
Flor & $21,78 \pm 4,43^{\mathrm{b}}$ & $1,27 \pm 0,35^{\mathrm{a}}$ & $6,35 \pm 2,25^{\mathrm{b}}$ \\
Botón Floral & $4,10 \pm 0,82^{\mathrm{a}}$ & $1,87 \pm 0,40^{\mathrm{a}}$ & $0,00 \pm 0,00^{\mathrm{a}}$ \\
Cápsula & $4,98 \pm 1,79^{\mathrm{a}}$ & $5,10 \pm 0,81^{\mathrm{b}}$ & $0,25 \pm 0,25^{\mathrm{a}}$ \\
\hline
\end{tabular}

Tabla 2. Abundancias ( $\pm \mathrm{SE}$ ) de las especies $T$. palmi, $S$. dorsalis y $F r$. cephalica en hoja joven, flor, botón floral y cápsula del cultivo de algodón en el valle cálido del alto Magdalena.

\begin{tabular}{lcccc}
\hline \multicolumn{1}{c}{ Especie } & Hoja & Flor & Botón floral & Cápsula \\
\hline T. palmi & $29,05 \pm 6,27^{\mathrm{c}}$ & $21,78 \pm 4,43^{\mathrm{c}}$ & $4,10 \pm 0,82^{\mathrm{b}}$ & $4,98 \pm 1,79^{\mathrm{b}}$ \\
S. dorsalis $^{\mathrm{b}}$ & $6,03 \pm 1,22^{\mathrm{b}}$ & $1,27 \pm 0,35^{\mathrm{a}}$ & $1,87 \pm 0,40^{\mathrm{b}}$ & $5,10 \pm 0,81^{\mathrm{c}}$ \\
Fr. cephalica & $0,15 \pm 0,09^{\mathrm{a}}$ & $6,35 \pm 2,25^{\mathrm{b}}$ & $0,00 \pm 0,00^{\mathrm{a}}$ & $0,25 \pm 0,25^{\mathrm{a}}$ \\
\hline
\end{tabular}

(Fig. 6). En el microhábitat flor, predominan Fr. cephalica y T. palmi; mientras que, en hoja, botón floral y cápsula, prevalecen S. dorsalis y T. palmi. Esta última, presentó los mayores índices de preferencia con respecto a Fr. cephalica y $S$. dorsalis. La comparación entre microhábitat para las especies presentó diferencias significativas en hoja joven $(\mathrm{H}=50,29 ; \mathrm{P}<0,0001)$, flor $(\mathrm{H}=35,52 ; \mathrm{P}<0,0001)$, botón floral $(\mathrm{H}=24,16 ; \mathrm{P}<0,0001)$ y cápsula $(\mathrm{H}=32,31 ; \mathrm{P}<$ 0,0001 ) (Tabla 2).

\section{Discusión}

En el cultivo de algodón Gossypium hirsutum L., uno de los cultivos de mayor importancia económica a nivel mundial, los trips de los géneros Caliothrips Daniel, Retithrips Marchal, Sericothrips Haliday, Thrips Linneaeus, Frankliniella Karny y Scirtothrips Shull, constituyen limitantes fitosanitarios claves en la producción (ThripsWiki 2016), con varias especies polífagas y hábito alimenticio fitófago con distri- 
A
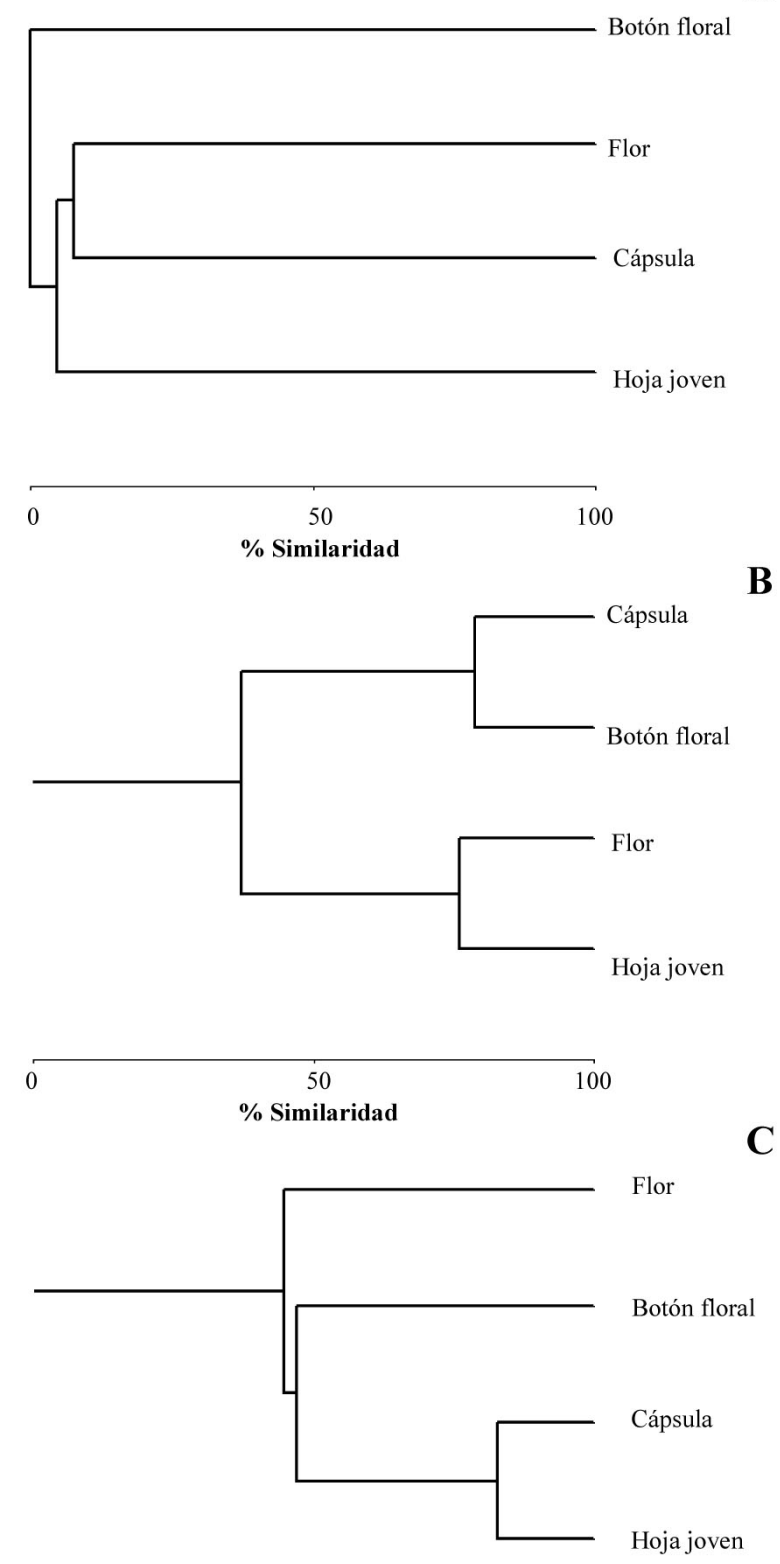

0

\section{0}

$\%$ Similaridad

Figura 5. Análisis de agrupamiento de Bray-Curtis entre los microháhitats del cultivo de algodón y las especies de trips estudiadas. A. T. palmi. B. S. dorsalis. C. Fr. cephalica.

bución en las zonas tropicales y subtropicales del mundo (Mound y Marullo 2012).

La herbivoría de T. palmi, S. dorsalis y Fr. cephalica en las estructuras de las plantas de algodón, muestra una relación de competencia interespecífica negativa o antibiosis por los recursos cápsula, botón floral, hoja joven y flor. Sin embargo, T. palmi, presentó una mayor amplitud y capacidad de explotación de los recursos, con niveles de abundancia superiores a $S$. dorsalis en hoja joven, botón floral y cápsula; y a Fr. cephalica en flor. Ante la presencia de especies invasoras como T. palmi y $S$. dorsalis en cultivos de algodón, las poblaciones de Fr. cephalica podrían resultar excluidas de las

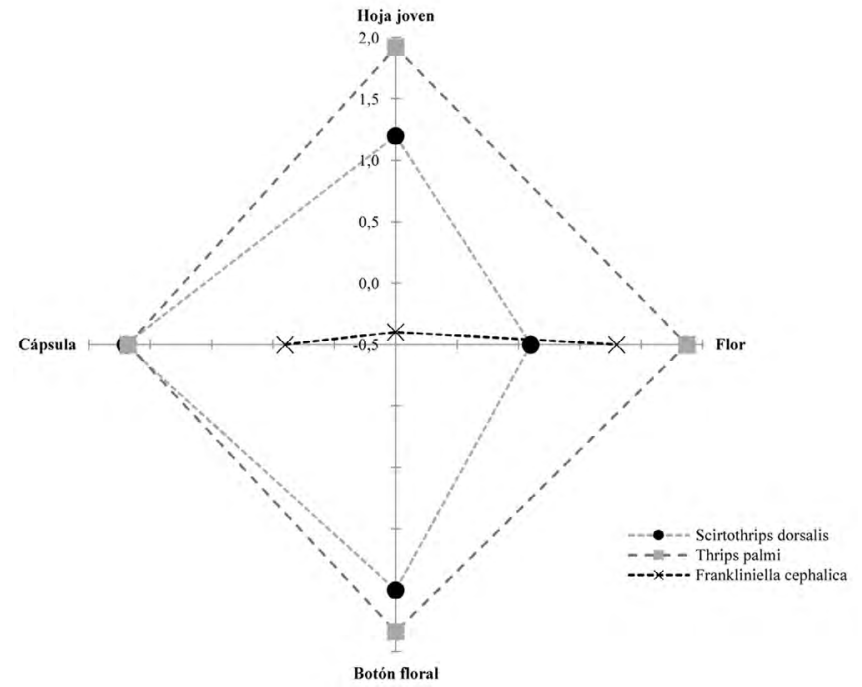

Figura 6. Interacción entre las especies de trips en el cultivo de algodón de acuerdo con los niveles de preferencia por los microhábitats.

flores y ser presionadas hacia una diferenciación de nicho, o una extinción local. En este caso, se evidenciaría el efecto de especies invasoras sobre especies indígenas como $\mathrm{Fr}$. cephalica en los agroecosistemas, pese a que la competencia es una interacción biológica en la cual la aptitud o adecuación biológica de una especie es reducida como consecuencia de la presencia de otra. En perjuicio de todas las especies involucradas, la competencia interespecífica es solo uno de los muchos factores bióticos que interactúan afectando la estructura de las comunidades ecológicas, al reducir la fecundidad, el crecimiento y la supervivencia de al menos una de las especies involucradas y puede cambiar las poblaciones, comunidades y la evolución de las especies que interactúan. Este panorama es aún más dramático y acelerado cuando entre las especies que compiten, se encuentran especies invasoras y nativas (Silveira-Neto et al. 1976; Zwolfer 1983; Liebhold et al. 1995; Liebhold y Tobin 2008).

De acuerdo con este estudio, T. palmi tiene una relación de tipo filófaga-carpófaga-antófaga en el cultivo de algodón. Scirtothrips dorsalis presentó una relación de tipo filófagacarpófaga y Fr. cephalica, una relación de tipo antofagia por la preferencia exclusiva por el microhábitat flor, como la mayoría de especies dentro del género Frankliniella Karny (Mound y Marullo 1996). Los microhábitats seleccionados por S. dorsalis, (hoja joven, botón floral y cápsula) corresponderían a recursos alimenticios y reproductivos, debido a que no requieren de polen para reproducirse (Mound y Stiller 2011). Al contrario, $F r$. cephalica, necesita polen para una mayor producción de huevos convirtiéndose en un recurso alimenticio necesario para la reproducción (Tsai et al. 1996).

A través de este estudio, el algodón se consolida como una planta hospedante verdadera para T. palmi, S. dorsalis y $F r$. cephalica, debido a que en estudios realizados por Zamar y Neder (2012), se resaltó que las condiciones de planta hospedera, se basa en que los insectos se establecen, se alimentan y se reproducen. Esta condición se observó de manera conjunta con la presencia de estados inmaduros (huevos y estados larvarios) y adultos (De Borbón 2007). Las especies de trips polífagas como $T$. palmi y $S$. dorsalis, tienden a ser plagas, en comparación con aquellas de hábito alimenticio monófago u oligófago. La polifagia y el intervalo de hospedantes están 
relacionados con la disponibilidad de compuestos químicos presentes en la planta, o por la inusual flexibilidad del insecto en la elección de las fuentes de alimentación (Pérez-Contreras 1999; Bernays y Chapman 2007; Marullo 2009; Zamar y Neder 2012).

Las especies de trips reportadas en este estudio, podrían incidir negativamente en los rendimientos esperados y en la calidad de la fibra de algodón producida en Colombia, tanto por los daños directos como por los indirectos a través de la trasmisión de partículas virales. Thrips palmi, en algodón, se ha observado causando daño en tejidos de hojas que pueden deformarse y despedazarse (Bournier 1987; Leigh 1995). En Brasil, Goussain et al. (2015), reportaron por primera vez esta especie causando daño a cultivos de algodón, siendo catalogada como plaga potencial. En relación con virus, Jagtap et al. (2012) encontraron un porcentaje bajo (3-5\%) de transmisión del virus del rayado del tabaco (TSV), que causa la enfermedad del mosaico del algodón.

Scirtothrips dorsalis, es la especie con mayor tasa de daño y abundancia estacional de estados inmaduros y adultos en el algodonero, en comparación con 12 cultivos de importancia económica entre los que se incluyen aguacate y mango en el sur de Florida (Kumar et al. 2012). Asimismo, se ha reportado como una de las 13 especies más peligrosas y exóticas que amenazan la industria, a causa de las pérdidas económicas que puede generar en cultivos de fresa, maní, pimentón, arándanos y algodón (Kumar et al. 2013).

Frankliniella cephalica, no se ha encontrado causando un daño específico a la planta de algodón, sin embargo, se ha comprobado su capacidad de trasmitir virus en las plantas hospedantes (Ohnishi et al. 2006). El género Frankliniella y específicamente, Frankliniella tritici fue encontrada en el $10 \%$ de las flores de algodón como portador del hongo $F u$ sarium verticillioides agente causal del síndrome denominado cápsula dura o hardlock; sin embargo, la implementación de control con insecticidas redujo la incidencia de cápsula dura y el número de trips, con aumentos en los rendimientos (Mailhot et al. 2007).

Los adultos de muchas especies de Thrips y Frankliniella, cuando hay escasez de flores en sus hospederos, se pueden alimentar de plantas en etapa de floración que no son adecuadas (Chellemi et al. 1994) y de hojas jóvenes, microhábitats que según Funderburk et al. (2002) son una fuente más estable de alimento para el desarrollo de estados inmaduros. Estas estrategias de alimentación, permiten referirse a especies de estos géneros como especies oportunistas, dado que son capaces de explotar ambientes que se presentan de forma intermitente (Mound y Marullo 2002). Estos ambientes, son controlados por factores climáticos que afectan la respuesta de las infestaciones de trips hacia cada microhábitat del cultivo de algodón (Cook et al. 2013). Al respecto, se han encontrado correlaciones significativas positivas entre las poblaciones de trips con temperaturas máximas y negativas con humedades relativas en horas de la mañana en algodones transgénicos de India y Turquía (Sitaramaraju et al. 2010; Shivanna et al. 2011).

\section{Conclusiones}

Thrips palmi, Scirtothrips dorsalis y Frankliniella cephalica se encuentran asociadas al cultivo de algodón en el valle cálido del alto Magdalena de Colombia y su presencia podría ocasionar efectos negativos en la calidad de la fibra y los rendimientos esperados. Son necesarios estudios sobre la biología, ecología, bioeconomía, generación de reglas de decisión como umbrales de acción y niveles de daño económico, en conjunto con estrategias de manejo integrado que permitan reducir la abundancia, dispersión y colonización de las poblaciones de trips y la capacidad de daño al cultivo de algodón.

\section{Agradecimientos}

Los autores agradecen a las diferentes agremiaciones del sector algodonero en los departamentos del Tolima, Huila y Cundinamarca, al Ministerio de Agricultura y Desarrollo Rural (MADR) por la financiación, a la Corporación Colombiana de Investigación Agropecuaria AGROSAVIA, al Instituto Colombiano Agropecuario ICA, la Universidad del Tolima, BIOQUALITYAGRO, la Universidad Nacional de Colombia con sede en Bogotá y la Ing. en Agroecología Jessica Vaca U., por sus observaciones, comentarios y decidida colaboración en la realización de esta publicación. Al doctor Arturo Goldarazena por su ayuda en la identificación de especímenes.

\section{Literatura citada}

ARÉVALO, P. E.; CORREA, L. G. 2003. Survey of thrips (Insecta: Thysanoptera) in flower crops at three localities of the municipality of Medellín, Antioquia (Colombia). Revista Colombiana de Entomología 29 (2): 169-175.

BERNAYS, E. A.; CHAPMAN, R. F. 2007. Host-plant selection by phytophagous insects (Vol. 2). Springer Science \& Business Media. $312 \mathrm{p}$.

BOURNIER, J. P. 1987. About the distribution of the noxious Thrips palmi Karny. En: Holman J. Population structure, genetics and taxonomy of aphids and Thysanoptera. Proceedings of International Symposium on Thysanoptera, Smolenice, Czechoslovakia, September 9-14, 1985.

BUENO, J. M.; CARDONA, C. 2003. Umbral de acción para Thrips palmi (Thysanoptera: Thripidae) en habichuela en el Valle del Cauca, Colombia. Revista Colombiana de Entomología 29 (1): 51-55.

CHELLEMI, D. O.; FUNDERBURK, J. E.; HALL, D. W. 1994. Seasonal abundance of flower-inhabiting Frankliniella species (Thysanoptera: Thripidae) on wild plant species. Environmental Entomology 23 (2): 337-342.

Confederación Colombiana del Algodón (CONALGODÓN). 2015. CONALGODÓN. 2016. Estadísticas. Disponible en: http://conalgodon.com/wp-content/uploads/2015/12/201507-InformeCosecha-Nacional.pdf. [Fecha revisión: 30 septiembre 2016].

COOK, D. R.; LEONARD, B. R.; BURRIS, E.; GORE, J. 2013. Impact of thrips infesting cotton seedlings on cotton yield distribution and maturity. Journal of Cotton Science 17: 23-33.

DE BORBÓN, C. M. 2007. Clave para la identificación del segundo estadio larval de algunos trips comunes (Thysanoptera: Thripidae) en Mendoza, Argentina. Revista de la Facultad de Ciencias Agrarias Universidad de Cuyo 39 (1): 69-81.

DI RIENZO, J. A.; CASANOVES, F.; BALZARINI, M. G.; GONZALEZ, L.; TABLADA, M.; ROBLEDO, C. W. 2016. Grupo InfoStat, FCA, Universidad Nacional de Córdoba, Argentina. Disponible en: http://www.infostat.com.ar. [Fecha revisión: 01 junio 2018].

ESPINEL, C.; TORRES, L.; GRIJALBA, E.; VILLAMIZAR, L.; COTES, A. M. 2008. Preformulados para control de la mosca blanca Bemisia tabaci (Hemiptera: Aleyrodidae) en condiciones de laboratorio. Revista Colombiana de Entomología 34 (1): 22-27.

European Food Safety Authority (EFSA). 2014. Scientific opinion on the pest categorization of Scirtothrips dorsalis EFSA panel on plant health (PLH). EFSA Journal 12 (12): 3915. 
FUNDERBURK, J.; STAVISKY, J.; TIPPING, C.; GORBET, D.; MOMOL, T.; BERGER, R. 2002. Infection of Frankliniella fusca (Thysanoptera: Thripidae) in peanut by nematode Thripinema fuscum (Tylenchidae: Allantomenatidae). Enviromental Entomology 31 (3): 558-563.

GOUSSAIN, M.; SILVEIRA, L. P.; HASS, J.; SANTO, R.; BALISTA, F.; CLARO, M.; ZUCONELLI, V.; MARCHIORO, L.; AGUILLERA, L. A. 2015. Primeiro relato de Thrips palmi Karny, 1925 (THRIPIDAE, THRIPINAE) atacando a cultura do algodão. $10^{\circ}$ Congreso Brasileiro do algodão. Disponible en: http://www.congressodoalgodao.com.br/livro-de-resumos2015/ resumos/R0548-1.html. [Fecha revisión: 10 enero 2016].

HODDLE M. S.; MOUND, L. A. 2003. The genus Scirtothrips in Australia (Insecta, Thysanoptera, Thripidae). Zootaxa 268 (1): 40.

JAGTAP, G. P.; JADHAV, T. H.; UTPAL, D. 2012. Host range and transmission of tobacco streak virus (TSV) causing cotton mosaic disease. Scientific Journal of Veterinary Advances 1 (1): 22-27.

JONES, D. R. 2005. Plant viruses transmitted by thrips. European Journal of Plant Pathology 113 (2): 119-157. Doi: doi. org/10.1007/s10658-005-2334-1.

KUMAR, V. G.; SEAL, D. R.; KAKKAR, G.; MCKENZIE, C. L.; OSBORNE, L. S. 2012. New tropical fruit hosts of Scirtothrips dorsalis (Thysanoptera: Thripidae) and its relative abundance on them in south Florida. Florida Entomologist 95 (1): 205-207.

KUMAR, V. G.; KAKKAR, C.; MCKENZIE, D. R.; OSBORNE, L. S. 2013. An Overview of Chilli Thrips, Scirtothrips dorsalis (Thysanoptera: Thripidae) Biology, distribution and management. En: Weed and pest control - Conventional and new challenges. Disponible en:_http://www.intechopen.com/books/ weed-and-pest-control-conventional-and-new-challenges/anoverview-of-chilli-thrips-scirtothrips-dorsalis-thysanopterathripidae-biology-distribution-and-m\#article-front. [Fecha revisión: 21 junio 2016].

LEIGH, T. F. 1995. Bionomics of cotton thrips: A review. pp. 6170. In: Thrips biology and management. Springer, Boston, MA. EE. UU.

LIEBHOLD, A. M.; MacDONALD, W. L.; BERGDAHL, D.; MASTRO, V. C. 1995. Invasion by Exotic Forest Pests: A Threat to Forest Ecosystems. Forest Science Monographs 30: 49.

LIEBHOLD A. M.; TOBIN P. C. 2008. Population ecology of insect invasions and their management. Annual Review Entomology 53: 387-408.

MAILHOT, D. J.; MAROIS, J. J.; WRIGHT, D. L. 2007. Influence of flower thrips on Fusarium hardlock severity. Plant Disease 91 (11): 1423-1429.

MARTÍNEZ, L.; QUINTANA J., E. M.; HERRERA C., R. F. 2003. Potencial de daño de Anthonomus grandis Boheman en el cultivo del algodonero en el Sinú medio. Temas Agrarios 8 (1): 7-10.

MARULLO, R. 2009. Host-plant ranges and pest potential: habits of some thrips species in areas of southern Italy. Bulletin of Insectology 62 (2): 253-255.

McALEECE, N.; GAGE, J. D. G.; LAMBSHEAD, P. J. D.; PATERSON, G. L. J. 1997. BioDiversity Professional statistics analysis software. Scottish Association for Marine Science y the Natural History Museum London.

MOUND, L. A.; MARULLO, R. 1996. The thrips of Central and South America: An introduction. Memoirs on Entomology International 6, $488 \mathrm{p}$.

MOUND, L. A.; MARULLO, R. 2002. Ecology of thrips. Thrips and Tospoviruses, pp. 121-128: Proceedings of the 7th International Symposium on Thysanoptera, Universita degli studi Mediterranea di Reggio Calabria. Facolta di Agraria. Dipartimento di Agrochimica ed Agrobiologia. Canberra: Australian National Insect Collection CSIRO.

MOUND, L. A.; MARULLO, R. 2012. Thysanoptera (Thrips) of the world - a checklist. Disponible en: http://www.ento.csiro.au/ thysanoptera/worldthrips.html. [Fecha revisión: 09 enero 2016].
MOUND, L. A.; STILLER, Y. M. 2011. Species of the genus Scirtothrips from Africa (Thysanoptera, Thripidae). Zootaxa 2786: 51-61.

OHNISHI, J.; KATSUZAKI, H.; TSUDA, S. 2006. Frankliniella cephalica, a new vector for Tomato spotted wilt virus. Plant Disease 90 (5): 685.

PÉREZ-CONTRERAS, T. 1999. La especialización en los insectos fitófagos: Una regla más que una excepción. Boletín Sociedad Entomología Aragonesa 26: 759-776.

ROMERO, L.; ARÉVALO, H.; DUARTE, W.; MEJÍA, R. 2012. Susceptibilidad de Heliothis virescens (Lepidoptera: Noctuidae) a la proteína Cry1Ac incorporada a dietas merídicas. Revista UDCA Actualidad \& Divulgación Científica 15 (2): 381-389.

SANTOS-AMAYA, O.; DELGADO-RESTREPO, O.; ARGÜELLES, J.; AGUILERA-GARRAMUÑO, E. 2009. Evaluación del comportamiento del complejo Spodoptera con la introducción de algodón transgénico al Tolima, Colombia. Revista Corpoica. Ciencia y Tecnología Agropecuaria 10 (1): 24-32.

SANTOS O., A.; VARÓN D., E. H.; GAIGL, A.; FLORIANO, A. 2012. Nivel de daño económico para Neohydatothrips signifer (Thysanoptera: Thripidae) en maracuyá en el Huila, Colombia. Revista Colombiana de Entomología 38 (1): 23-29.

SARABIA, M. G.; CABALLERO, H. M.; CANO, P. S. 2015. Trips (Thysanoptera) del racimo del banano y sus enemigos naturales en el departamento del Magdalena, Colombia. Temas Agrarios 20 (2): 72-80.

SHIVANNA B. K.; GANGADHARA, B.; BASAVARAJA, M. K.; NAGARAJA, R.; KALLESWARA, C. M.; KAREGOWDA, C. 2011. Impact of abiotic factors on population dynamics of sucking pests in transgenic cotton ecosystem. International Journal of Natural Sciences 2: 72-74.

SITARAMARAJU, S.; PRASAD, N. V. V. S. D.; KRISHNAIAH, P. V. 2010. Seasonal incidence of sucking insect pests on Bt cotton in relation to weather parameters. Annals of Plant Protection Sciences 18 (1): 49-52.

SILVEIRA-NETO, S.; NAKANO, O.; VILLA NOVA, N. A. 1976. Manual de ecología dos insectos. 15th edition.- Agronômica Ceres, Piracicaba, SP, Brasil. 420 p.

THRIPSWIKI. 2016. Thripidae. Disponible en: http://thrips.info/w/ index.php?title $=$ Thripidae $\&$ oldid $=33251$. [Fecha revisión: 09 enero 2016].

THRIPSWIKI. 2018. Thripidae. Disponible en: https://thrips.info/ wiki/Thripidae. [Fecha revisión: 29 mayo 2018].

TSAI, J.; YUE, B.; FUNDERBURK, J.; WEBB, S. 1996. Effect of plant pollen on growth and reproduction of Frankliniella bispinosa. Tospoviruses and Thrips of Floral and Vegetable Crops 431: 535-541.

ZAMAR, M. I.; NEDER, L. E. 2012. Asociación Thysanoptera (Insecta)-Vicia faba (Fabaceae) en la Prepuna y Puna de Jujuy, Argentina. Revista de Biología Tropical 60 (1): 119-128.

ZWOLFER, H. 1983. Life systems and strategies of resource exploitation in tephritids. pp. 16-30. Cavalloro, R. (Ed.). Fruit flies of economic importance. Commission of the European communities. International Organization for Biological Control. A.A. Balkema Publishers. Rotterdam, Países Bajos. 642 p.

Recibido: 04-abr-2017 • Aceptado: 04-jul-2018

Citación sugerida:

JARAMILLO-BARRIOS, C.; RODRÍGUEZ, A.; VARÓN-DEVIA, E.; MONJE-ANDRADE, B.; EBRATT-RAVELO, E. 2018. Preferencias de tisanópteros (Thysanoptera) por las estructuras aéreas de las plantas de algodón (Gossypium hirsutum) en Colombia. Revista Colombiana de Entomología 44 (2): 151-157. Julio - Diciembre 2018. 